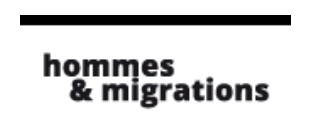

Hommes \& migrations

Revue française de référence sur les dynamiques

migratoires

$1300 \mid 2012$

Nouveaux modèles migratoires en Méditerranée

\title{
Métiers domestiques, voile et féminisme
}

Nouveaux objets, nouvelles ruptures

\section{Nasima Moujoud}

\section{OpenEdition}

1 Journals

\section{Édition électronique}

URL : http://journals.openedition.org/hommesmigrations/925

DOI : 10.4000/hommesmigrations.925

ISSN : 2262-3353

Éditeur

Musée national de l'histoire de l'immigration

\section{Édition imprimée}

Date de publication : 1 novembre 2012

Pagination : 84-94

ISSN : 1142-852X

\section{Référence électronique}

Nasima Moujoud, « Métiers domestiques, voile et féminisme », Hommes \& migrations [En ligne],

1300 | 2012, mis en ligne le 01 novembre 2014, consulté le 19 avril 2019. URL : http://

journals.openedition.org/hommesmigrations/925; DOI : 10.4000/hommesmigrations.925

Tous droits réservés 


\section{Métiers domestiques, voile et féminisme Nouveaux objets, nouvelles ruptures}

Par Nasima Moujoud, maître de conférences en anthropologie, LARHRA-université-Pierre Mendès France

En France, le travail des migrantes souffre d'un déficit d'analyse. Si la recherche féministe prend bien pour objet la position des femmes immigrées, elle a tendance à oublier les dynamiques profondes qui déterminent leur histoire. L'insertion de ces femmes dans les métiers de service et l'affirmation de leur identité culturelle à travers le port du voile ne sont pas des phénomènes récents. Pour les comprendre, il faut savoir décrypter les mécanismes de racialisation à l'œuvre au sein de la société française. 
La production théorique féministe française qui aborde la migration à partir du début des années 2000 affirme travailler de façon critique sur des dominations multiples et imbriquées. Elle se définit en dialogue avec des problématiques anglosaxonnes et ignore très souvent le travail théorique fondateur effectué par des spécialistes qui ont défini les champs de recherche sur la migration, la colonisation ou le racisme en France et dans le contexte francophone. Le principal trait commun de cette production est l'occultation de l'histoire coloniale. Ce trait conduit à ignorer l'histoire postcoloniale de la migration ainsi que les conditions d'origine de "ses" émigré(e)s, ce qui, selon Abdelmalek Sayad, condamne toute recherche "à ne donner du phénomène migratoire qu'une vision partielle et ethnocentrique $e^{(1)}$ ".

Deux objets tirés de travaux récents ayant mis le travail des femmes au centre de leurs théories sur la société française "en général" peuvent aider à rendre compte de ces aspects : les métiers domestiques, souvent reliés à la mondialisation, et le voile, qui a été central dans l'émergence d'un débat féministe sur sexisme, racisme et/ou multiculturalisme en France depuis, notamment, 2004. Ainsi, après avoir précisé l'émergence récente de la migration dans la recherche féministe majoritaire, j'essaierai de montrer que son occultation de l'histoire coloniale éclaire l'ignorance de l'ensemble des impacts induits par la colonisation comme de l'histoire de la migration en France ${ }^{(2)}$. On verra que les analyses d'Abdelmalek Sayad, qui a invité à la fois à ne pas réduire l'étude de la migration à la société d'immigration et à ne pas considérer les sociétés dites "sous-développées" comme précapitalistes, pourraient bien s'appliquer aux travaux féministes majoritaires récents que la vision binaire des sociétés n'en finit pas d'influencer.

\section{Après le silence}

En France, alors que le débat, les pratiques et les lois touchant les migrants remontent à plus d'un siècle, la migration n'a pas été initialement intégrée dans les théories féministes sur la société française ${ }^{(3)}$. La fermeture du féminisme français aux problématiques migratoires est soulignée au Québec, où Chantal Maillé précise que le retard du féminisme local tient en partie à "l'influence du féminisme français, relativement fermé aux débats qui ont émergé dans d'autres milieux féministes (4)" $^{\prime}$.

Tandis que la migration est depuis longtemps reconnue comme un phénomène structurel des sociétés capitalistes, et que les théories féministes ont largement mis en évidence le lien entre modes de production et de reproduction sociales ${ }^{(5)}$, la migration a pendant longtemps été occultée par les théories féministes " générales ". Malgré la prégnance des théorisations féministes dans les années 1970, bien peu de questionne- 
ments se sont consacrés à la position des migrantes, tandis que la dimension juridique est restée essentiellement réduite à celles des "Françaises". Pourtant, il est évident que l'impacts des lois n'est pas le même pour une femme autochtone et pour une femme migrante et, très souvent, issue de milieux populaires. Par exemple, les théories féministes des années 1970 et 1980 ne se saisissent pas des mesures encadrant le travail des migrantes en France, comme la mesure prise le 10 novembre 1977 qui prohibe la délivrance d'un titre de travail aux membres des familles entrées après cette date ${ }^{(6)}$. Plus tard, dans les années 1990, malgré la surreprésentation des migrantes dans les emplois familiaux, dont les politiques ont essuyé de nombreuses critiques féministes, bien peu de réflexions se sont consacrées à la division raciale du travail entre les femmes.

Pourtant, en France, les études "sur les femmes immigrées" ont débuté réellement à partir de la deuxième partie des années 1970 dans un champ à part, qui s'est forcément appuyé sur les théories féministes. Il ne concerne pas toutes les femmes susceptibles de s'autodésigner ou d'être perçues comme migrantes et s'est largement focalisé sur la migration féminine postcoloniale, limitée au cadre familial et très souvent appréhendée sous l'angle de lectures assimilées au Maghreb et évitant de voir le travail des femmes ${ }^{(7)}$.

\section{Construire une perspective critique postcoloniale}

La thématique "travail" a pourtant été au centre de la recherche sur l'immigration (des "(hommes) travailleurs immigrés") comme dans la recherche féministe sur la société française "en général". Le travail est un thème central dans le féminisme. Les théories féministes matérialistes ont montré que la division sexuelle du travail construisait à la fois les "femmes" et les "hommes" et l'ensemble de l'organisation sociale. Elles ont mis en évidence la vision androcentrique du concept de travail qui a mené à occulter le travail des femmes, l'activité économique étant définie et pensée en référence au seul travail productif et autour de la figure de l'homme travailleur. Or, si cette dernière idée semble relativement évidente, notamment dans les travaux féministes sur "les sociétés industrielles", des catégories réductrices empêchent les auteurs de ces travaux de le voir pour les sociétés dites "sous-développées" et, par conséquent, pour les sociétés ex-colonisées et les groupes migrants issus de ces sociétés. Nombreuses sont les féministes spécialistes du travail qui, peut-être pour ne pas risquer des comparaisons mal étayées, disent s'en tenir à l'étude des "sociétés industrialisées". Par exemple, Helena Hirata et Danième Kergoat précisent que leur analyse sur la division sexuelle du travail porte sur les sociétés salariales comme "sociétés dites ' $d u$ 
Nord ${ }^{p(8)}$; Monique Haicault indique que sa théorie concerne uniquement les "sociétés industrielles "(9)". Comme dans d'autres points de vue représentant des théories "générales", ces auteures n'interrogent ni la catégorie des sociétés qu'elles désignent ni les raisons de leur limitation. Elles partent d'un biais épistémologique implicite qui consiste à tenir pour acquise l'unité présumée d'un ensemble (l'Occident) par opposition à un autre ensemble (le Reste du monde), tandis que les continuités entre ces deux ensembles construits sont masquées.

Pourtant, le discours fondé sur l'opposition entre deux ensembles de sociétés renvoie au contexte colonial ${ }^{(10)}$. Celui-ci a rendu particulièrement vivante l'opposition tradition/modernité, doublée de celle entre Occident et non-Occident, qui

\section{Les nouvelles approches féministes évoquent la migration de femmes du Sud qui partiraient vers le Nord pour remplacer des femmes autochtones qui accèdent au travail salarié et désirent se libérer des travaux domestiques.} donne lieu, entre autres, à des discours orientalistes dont les effets sexués sont fortement critiqués dans le féminisme postcolonial ${ }^{(11)}$. Parallèlement, l'opposition entre deux catégories de sociétés oublie que la colonisation a été le principal outil d'expansion du capitalisme. Les "autres" sociétés ont donc depuis maintenant relativement longtemps fait l'expérience du rapport entre reproduction et mode de production salariée, comme le montrent les études sur la façon dont l'histoire coloniale a transformé les rapports sociaux de sexe ${ }^{(12)}$. À force de négliger les champs de recherche sur la colonisation et sur les "autres" sociétés, les théories féministes françaises "générales" donnent une fausse idée de la France et des sociétés ex-colonisées, ignorent les continuités en somme. En même temps, ces théories découvrent aujourd'hui la migration en oubliant son histoire coloniale.

C'est à partir du début des années 2000 que se développent dans la recherche féministe des dossiers sur les migrations (comme les Cahiers du CEDREF n ${ }^{\circ} 8 / 9$, 2000), des numéros spécialisés ne portant pas sur le cas français (Nouvelles questions féministes, vol. 26, n 1,2007) et des travaux féministes se référant soit à la thématique des emplois domestiques, soit à celle du racisme (Nouvelles questions féministes, 2006) et comportant des interrogations sur les dominations imbriquées (sexe, "race", classe...). L'émergence de la question migratoire se développe au moment où le féminisme majoritaire se définit comme "l'un des rares champs de recherche à affronter avec autant de perspicacité et de probité intellectuelle l'analyse des mécanismes entremêlés de la domination ${ }^{(13)}$. Or, paradoxalement, cette analyse ignore, entre autres, le travail théorique fondateur effectué par des théoriciens français/francophones, tels Abdelmalek Sayad et Fatima Mernissi, qui ont pourtant contribué de façon centrale à rendre possible une 
analyse critique des rapports sociaux de pouvoir et des modes de production datant de la colonisation. Elle reconduit ainsi la rupture avec les "anciens" champs de recherche sur la migration et oublie, entre autres, le lien entre migration et colonisation.

\section{Les "nouveaux" métiers domestiques}

Le travail des femmes (ex-)colonisées et des migrantes est resté en marge des champs d'étude de l'histoire de la migration comme de la colonisation ou de la division raciale du travail, tout autant que de l'histoire du travail des femmes et des théories féministes sur la division sexuelle du travail. Pourtant, il possède en France une longue histoire qui conduit à interroger les limites des nouvelles approches féministes des métiers domestiques.

Le silence féministe a historiquement marqué le sujet des métiers domestiques en général, comme le note Geneviève Fraisse qui distingue les féministes qui ont abordé le travail domestique (gratuit des femmes) et les "sociologues en général" qui "réfléchissent sur l'emploi de service ${ }^{(14) "}$. À partir de 2000, la question des métiers domestiques émerge dans des travaux de féministes dont les théories ont initialement mis en évidence la centralité du travail dans l'oppression des femmes en France "en général". La principale entrée dans ces travaux est la "crise de la reproduction sociale" dans le contexte de la mondialisation. Pour Helena Hirata, comme pour d'autres auteurs qu'elle cite, une des conséquences de la mondialisation est 1'“internationalisation $d u$

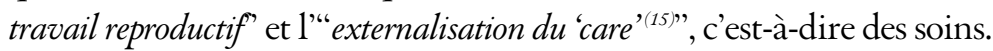

Les nouvelles approches féministes évoquent la migration de femmes du Sud qui partiraient vers le Nord pour remplacer des femmes autochtones qui accèdent au travail salarié et désirent se libérer des travaux domestiques. La migration de femmes orientées vers les services est ainsi placée au cceur de la mondialisation actuelle, des mutations que connaissent les "femmes du Nord" et des critiques des lectures dominantes sur la mondialisation. Cependant, cette migration est vue seulement dans le sens Sud/Nord, et non pas Sud/Sud. Si les mutations paraissent centrales au Nord, elles ne sont pas appréhendées du côté des "autres" sociétés, dont les émigrées sont ainsi considérées comme une catégorie de femmes à part, distinguées des "femmes du Nord". De nouveau, les sociétés se retrouvent perçues à travers des dichotomies qui masquent les continuités et les rapports historiques.

La migration des "femmes du Sud" est assimilée à la seule mondialisation actuelle. Très souvent, les nouvelles approches féministes majoritaires ne la situent pas dans le contexte français ou dans le cadre de l'histoire de chaque société d'origine et de ses relations avec la France. Cette limite conduit à l'idée que l'apparition de femmes 
prolétaires du Sud daterait de la mondialisation, comme dans le texte de Ribas-Mateos et Manry ${ }^{(16)}$, ou à celle de la nouveauté de la racialisation des emplois domestiques, comme dans le texte de Danièle Kergoat ${ }^{(17)}$, qui a par ailleurs prôné la nécessaire historicisation des rapports de pouvoir.

\section{Des emplois de service racialisés}

Danièle Kergoat relève "l'enjeu que le travail du care représente dans les sociétés occidentales" en l'assimilant à un "phénomène nouveau ${ }^{(18) "}$. Ce phénomène n'est situé ni dans le cadre de l'histoire des services domestiques ni dans l'histoire de la migration en France. Il n'est pas situé non plus dans des trajectoires et des histoires migratoires nationales ou régionales. Les métiers domestiques effectués par les "autres" femmes se retrouvent ainsi placés dans un contexte global incertain qui concernerait seulement, de la même manière et au même moment, les sociétés dites "occidentales" : les nouveaux pays d'immigration, comme l'Espagne et l'Italie qui connaissent par ailleurs une visibilité politique, statistique et scientifique de ces métiers, seraient concernés au même titre que les anciens pays d'immigration, comme la France qui a ses propres catégories scientifiques, ses politiques migratoires et sa propre histoire de recrutement de la main-d'ceuvre étrangère.

Certes, les ambiguïtés des politiques françaises qui sollicitent et invisibilisent en même temps le travail de femmes migrantes et/ou racialisées peuvent être en partie responsables des cécités de la recherche féministe majoritaire sur le sujet, comme nous avons pu le préciser dans un autre travail ${ }^{(19)}$. Or ces politiques ne sont pas inexistantes : par exemple, dans les années 1960 et 1970, des femmes antillaises font l'objet de politiques de recrutement à travers le Bumidom qui les destine aux métiers de service en métropole. 45 \% des Antillaises venues entre 1962 et 1968 entrent dans la catégorie "personnels de service" (12\% femmes de ménage et 33 \% autres personnel de service). Ces femmes vont faire usage de leur nationalité française pour s'orienter vers d'autres secteurs de travail, comme le montre Stéphanie Condon, dont le travail permet d'historiciser la racialisation des métiers domestiques en France ${ }^{(20)}$. Ensuite, les politiques migratoires peuvent rendre certaines femmes statistiquement absentes, car ne possédant pas les papiers français nécessaires, les pousser vers les secteurs à fort taux d'activité clandestine (domesticité, habillement, confection), mais, en même temps, les visibiliser lors de procédures de régularisation, comme en 1981-1984 où les activités de services concentrent plus de $40 \%$ des femmes régularisées ${ }^{(21)}$.

Les cécités de la recherche féministe majoritaire peuvent s'expliquer par sa tendance à éclipser les travaux de spécialistes qui se sont historiquement centrés sur la migration 
en France. En effet, la féminisation de la migration, son rajeunissement, l'accélération du regroupement familial, ainsi que l'orientation des femmes vers les métiers domestiques font partie des principales évolutions de la deuxième moitié des années 1960, ainsi que le montraient déjà en 1982 Yann Moulier et Roxane Silberman en notant les obstacles d'ordre juridique historiquement érigés contre l'emploi des immigrées. Elles insistent aussi sur la "montée de l'activité des femmes étrangères en France $^{(22) "}$ et le glissement de secteur de travail pour les migrantes légales (et donc statistiquement visibles), avec l'arrivée d'Africaines (dont les Maghrébines) et de Turques. En même temps, si en France, comme dans d'autres pays d'immigration, ainsi que le souligne Catherine Quiminal ${ }^{(23)}$, les recherches sur le genre et la migration sont souvent initiées par des femmes d'origine étrangère, nous ne trouvons généralement pas mention des travaux de ces auteures dans la recherche féministe française majoritaire qui s'est penchée récemment sur la migration en relation avec la question des dominations imbriquées ${ }^{(24)}$. Ainsi, la recherche féministe rend invisibles les groupes des femmes racialisées, migrantes ou descendantes de migrants. Les nouvelles approches critiques prennent en compte la question raciale mais l'inscrivent dans le cadre du nouveau débat entre féministes majoritaires.

\section{Le voile de la rupture}

Le savoir féministe majoritaire émergeant en France depuis 2004 se saisit amplement du voile. Ce "foulard de la dispute" a "provoqué de profonds déchirements parmi les féministes ${ }^{(25)}$ ". Les unes insistent sur la soumission des femmes et limitent la lecture du voile à la religion ou au contrôle du corps des femmes, sans situer les différentes positions dans le cadre de l'histoire coloniale; d'autres introduisent des nuances en démontrant la complexité des attitudes face au voile et au contrôle du corps des femmes. Ces dernières font souvent apparaître la dimension postcoloniale à travers l'impact des représentations héritées de la colonisation. Parmi elles, des chercheuses féministes mettent en évidence le lien entre racisme et sexisme et posent ainsi les termes d'un débat qui contribue à enrichir la réflexion sur le féminisme français. La question du voile se construit au centre de la problématique des dominations imbriquées. Cependant, si les écrits mettant en évidence le lien entre racisme et sexisme font voir l'impact du contexte politique français et permettent ainsi de sortir de l'islam, leur analyse crée deux ruptures principales avec, d'une part, la recherche sur l'histoire coloniale et/ou migratoire et, d'autre part, la recherche féministe "générale" qui a eu le mérite de démontrer la centralité du travail dans l'oppression des femmes ${ }^{(26)}$. La première rupture principale reconduite par le nouveau féminisme qui se saisit du 
voile est de laisser de côté les champs de recherche sur la colonisation, les sociétés excolonisées et/ou la migration. Cette rupture produit des visions réductrices qui apparaissent au niveau du regard sur "les autres" comme du regard sur le féminisme. Par exemple, dans l'analyse du lien entre féminisme, différence et multiculturalisme, ce lien est réduit à un phénomène récent qui limite la différence au voile, le voile à la différence et, en même temps, le féminisme aux féministes "occidentales" ou "françaises" majoritaires ${ }^{(27)}$. Parallèlement, les visions réductrices apparaissent au niveau de la prise en compte de l'histoire coloniale : lorsqu'elle est évoquée, celle-ci se limite souvent au vêtement et à l'idée de dévoilement imposé aux femmes pendant la guerre d'Algérie. Or, si la domination coloniale participe à l'imposition/acquisition de nouvelles normes, la période coloniale a été à la fois un moment de dévoilement et de voilement des femmes suivant leur âge, leur appartenance sociale, régionale, ethnique ou religieuse. En ce sens, Germaine Tillion

Tout laisse à penser
que les approches
majoritaires du "voile"
semblent considérer
que la dimension raciale
est récente, ne se situe
pas dans l'histoire
coloniale/migratoire
et révèle des mutations
uniques et nouvelles.
explique qu'en général le voile des Maghrébines est en relation avec l'urbanisation ${ }^{(28)}$. En même temps, le regard réducteur sur les "autres" influe sur la prise en compte du racisme, qui se réduit souvent à (l'idée de respect de) "la culture" des "autres". Sont privilégiés les aspects perçus comme "différents", dans une vision qui oppose les "autres" à un "nous" "occidental", "européen" ou "français". Pourtant, la différence culturelle a beaucoup à voir avec le regard colonial sur le Maghreb qui a rendu caractéristiques un certain nombre de pratiques en rapport avec le corps, le travail, la sexualité et la mobilité des femmes au Maghreb et en contexte migratoire. C'est ce que montrent Meriem Rodary et Simona Tersigni, qui mobilisent des approches postcoloniales, comme celles de l'anthropologie critique qui a insisté sur la nécessité de tourner le dos au culturalisme et aux conceptions des normes des "autres" comme fixées une fois pour toutes ${ }^{(29)}$.

\section{Dévoiler les continuités historiques}

Les nouvelles approches féministes du voile rompent également avec l'histoire de la recherche féministe "générale". Les deux lectures, favorables ou opposées au voile, s'écartent des théories féministes, notamment matérialistes, qui insistent sur les deux faces idéologiques et matérielles de l'oppression des femmes. Aussi, le travail disparaîtil dans le débat sur le voile. Celui-ci se réduit souvent à des dimensions idéologiques 
qui situent "la différence" dans "la culture" sans se soucier des dimensions matérielles du vécu des femmes voilées/non voilées subissant le racisme et les discriminations indépendamment de l'âge, de la nationalité, du statut juridique, de "la culture" ou de la loi contre les signes religieux ${ }^{(30)}$. Les effets de l'invisibilisation des champs de recherche sur l'histoire coloniale ou migratoire se doublent d'une rupture avec les acquis des "anciennes" théories féministes. Aussi est-il important de faire un retour sur le lien entre voile, travail et histoire coloniale.

Le (non-)voile représente une catégorie d'infériorisation qui précède la colonisation et se redéfinit en cette période d'expansion du capitalisme. Par exemple, au Maroc, le voile a historiquement marqué le regard des hommes citadins (riches, arabophones et quantitativement minoritaires) sur les paysan(ne)s (pauvres, montagnard(e)s, arabophones ou amazigh(e)s). Ce regard lie le voile au travail. Les femmes qui "sortent sans voile et vont travailler à la forêt, aux pâturages et dans les champs à côté des bergers" attisent la critique d'un citadin du XVI siècle qui renvoie la responsabilité de cette "insolence (...) inouie sur les hommes ruraux ${ }^{(31)}$ ". En même temps, dans la deuxième moitié des années 1930, des Marocaines riches de la ville de Fès se mobilisent pour redéfinir leur vêtement et leur accès à la rue ${ }^{(32)}$. Leur "dévoilement" ne peut être réduit à l'instrumentalisation colonialiste. Voile et travail vont être utilisés dans le cadre du projet nationaliste bourgeois qui envisage la libération des femmes par le dévoilement et l'accès au travail (salarié). Celui-ci sera défini à travers des normes masculines, capitalistes et "modernes". Il ne valorise pas le travail des ouvrières et rend invisible le travail informel, agricole ou précaire des femmes populaires/paysannes qui "étaient dévoilées et travaillaient dans les champs du matin au soir comme elles l'ont fait pendant des siècles avant les nationalistes ${ }^{(33) " . ~ L a ~ p o r t e ~ d e ~ l a ~ m o d e r n i t e ́ ~ p o s t c o l o n i a l e ~ n e ~ s e r a ~ p a s ~ a i s e ́-~}$ ment ouverte aux paysannes qui seront assimilées à "la tradition" et, paradoxalement, au voile et au non-travail ${ }^{(34)}$.

Cependant, les (anciennes) paysannes ne seront pas empêchées de franchir les frontières régionales et nationales. À partir, notamment, du début des années 1970, elles seront majoritaires parmi les Marocaines en France. Leur migration obéit à des logiques similaires à celles analysées par Abdelmalek Sayad sur la migration masculine entre l'Algérie et la France : l'émigration-immigration est "fille" de la colonisation. Elle ne se comprend qu'en regard des conditions historiques, socioéconomiques et culturelles qui ont guidé ses flux pendant la colonisation. Aussi, l'histoire de l'émigration se confond-elle avec celle de la paysannerie et des dépossessions foncières ${ }^{(35)}$. Toutefois, les migrantes nord-africaines seront longtemps vues à travers des stéréotypes raciaux, culturels et sociaux, bien que soumises à des accords bilatéraux et à des politiques migratoires historiquement sexuées et souvent dépourvues de droits indépendants ${ }^{(36)}$. Par extension, leur histoire marque aussi le 
regard sur leurs descendantes bien qu'elles soient souvent nées en France et y aient varié les attitudes face aux assignations identitaires ${ }^{(37)}$.

En définitive, la survisibilisation par le voile ne correspond pas à la place marginale qu'occupent dans le féminisme théorique majoritaire les problématiques liées au travail, à l'égalité et à l'accès aux ressources des migrantes et de leurs descendantes (voilées, non voilées...) en France et dans les sociétés (ex-)colonisées. Tout laisse à penser que les approches majoritaires du "voile" semblent considérer - comme dans les approches sur les "nouveaux" métiers domestiques - que la dimension raciale est récente, ne se situe pas dans l'histoire coloniale/migratoire et révèle des mutations uniques et nouvelles. La recherche féministe ne peut continuer à pécher par omission de l'histoire (coloniale et migratoire) alors qu'elle se penche dorénavant sur le racisme et les dominations entremêlées. Elle gagnerait peut-être à faire un retour critique sur les "anciennes" théories féministes, afin de saisir les effets du processus de rupture avec la migration et la colonisation. Ce retour appelle une re-connaissance de cette histoire et des savoirs transnationaux qui lui sont liés.

\section{Notes}

1. Abdelmalek Sayad, La Double Absence. Des illusions aux souffrances de l'immigré, Paris, Seuil, 1999, p. 56.

2. Notons l'absence d'entrée "colonisation" ou "ethnocentrisme" dans le Dictionnaire critique du féminisme (Helena Hirata, Françoise Laborie, Hélène Le Doaré, Danièle Senotier [dir.]), Paris, PUF, 2000.

3. Il n'est bien sûr pas question ici de présenter un bilan complet de la question migratoire dans le féminisme, étant donné la diversité de ce dernier.

4. Chantal Maillé, "Migrations : femmes, mouvement et 'refondation' du féminisme", in Recherches féministes, vol. $15, \mathrm{n}^{\circ} 2,2002$, p. 2 .

5. Christine Delphy, L'Ennemi principal. Penser le genre, Paris, Syllepse, 2001 [1970].

6. Si l'on tient compte du fait qu'une grande majorité des personnes regroupées est de sexe féminin, il est possible de prévoir l'importance de l'impact des lois migratoires sur le travail ainsi que sur l'autonomie juridique des femmes en contexte migratoire. Voir Nasima Moujoud, Jules Falquet, "Cent ans de sollicitude en France. Domesticité, reproduction sociale, migration et histoire coloniale", in Agone, $\mathrm{n}^{\circ} 43,2010$.

7. Sabah Chaïb, "Femmes immigrées et travail salarié en France", in Jules Falquet et al., (dir.), Femmes, genre, migrations et mondialisation. Un état des problématiques, Cahiers du CEDREF, Paris, université Denis-Diderot, 2008 ; Meriem Rodary, "Le travail des femmes dans le Maroc précolonial, entre oppression et résistance. Droit au travail ou accès aux bénéfices?", in Cahiers d'études africaines, vol. XLVII (3-4), n 187-188, 2007, pp. 753-780.

8. Helena Hirata, Danièle Kergoat, "La division sexuelle du travail revisitée", in Marguaret Maruani, Les Nouvelles Frontières de l'inégalité. Hommes et femmes sur le marché du travail, Paris, La Découverte/Mage, 1998, p. 95.

9. Monique Haicault, "La gestion ordinaire de la vie à deux ", in Sociologie du travail, n³, 1984, pp. 268.

10. Les lignes du "grand partage" entre sociétés "traditionnelles, primitives" et "modernes, occidentales" sont mises en cause dans l'anthropologie critique (par exemple, Jack Goody en 1979).

11. Lila Abu-Lughud, "Feminist longings and postcolonial conditions", in Lila Abu-Lughod (dir.), Remaking Women. Feminism and Modernity in the Middle-East, Princeton, Princeton University Press, 1998, pp. 3-31.

12. Fatima Mernissi, "Développement capitaliste et perception des femmes dans la société arabo-musulmane: une illustration des paysannes du Gharb, Maroc", étude préparée pour le séminaire régional tripartite du BIT pour l'Afrique, 1981 ; Meriem Rodary, "Le travail des femmes dans le Maroc précolonial, entre oppression et résistance. Droit au travail ou accès aux bénéfices?", op. cit.

13. Elsa Dorlin, "Vers une épistémologie des résistances", in Elsa Dorlin (dir.), Sexe, race, classe. Pour une épistémologie de la domination, Paris, PUF, 2009, p. 5 . 
14. Geneviève Fraisse, "Domesticité, emploi de service et démocratie", in Margaret Maruani, op. cit., p. 155.

15. Helena Hirata, Danièle Kergoat, "La division sexuelle du travail revisitée", op. cit., p. 8.

16. Véronique Manry, Natalia Ribas-Mateos, "Mobilités au féminin, ou comment appréhender la place des femmes et des mobilités dans la globalisation", in Mobilités au féminin, colloque international, 15-19 novembre 2005, Tanger, sur www.mmsh.univ-aix.fr (consulté le 20 novembre 2005).

17. Danièle Kergoat, "Dynamique et consubstantialité des rapports sociaux", in Elsa Dorlin (dir.), op. cit., pp. 111-125.

18. Danièle Kergoat écrit : "ce rapport [de race] entre maintenant, par l'intermédiaire de la nounou, de la femme de ménage ou de la femme qui prend soin des membres très âgés de la famille - et c'est un phénomène nouveau -, au cceur même des familles. L'autre, l'étranger, n'est plus seulement à la périphérie des grandes villes, dans les bidonvilles ou dans les 'banlieues'. Il/elle vit et travaille au sein même de familles qui, il n'y a pas si longtemps, ne pouvaient être touchées par ce phénomène." Danièle Kergoat, "Dynamique et consubstantialité des rapports sociaux", op. cit., p. 122.

19. Nasima Moujoud, Jules Falquet, "Cent ans de sollicitude en France. Domesticité, reproduction sociale, migration et histoire coloniale", art. cit.

20. Stéphanie Condon, "Migrations antillaises en métropole : politique migratoire, emploi et place spécifique des femmes", in Cahiers du CEDREF, n 8/9, 2000, pp. 167-200.

21. Smaïn Laâcher, "L'État et l'Immigration clandestine. Entre la question du travail et celle des illégalismes, Rapport français, Commission européenne, Maison des sciences de lhHomme, 1998.

22. Moulier Silberman

23. Catherine Quiminal, "Migrations”, in Helena Hirata, Françoise Laborie, Hélène Le Doaré, Danièle Senotier (dir.), Dictionnaire critique du féminisme, op. cit., pp. 111-116.

24. Par exemple, Danièle Kergoat ne cite pas les spécialistes du sujet, comme Sabah Chaïb, une des fondatrices de la recherche sur genre, travail et migration en France ; Annette Goldberg Salinas, sur la place des migrantes dans la recherche féministe française ou Faïza Guélamine sur le racisme et l'héritage colonial. Abdelmalek Sayad ne trouve également pas place dans le travail de Danièle Kergoat comme dans le "nouveau" champ de recherche féministe qui se penche aujourd'hui sur racisme, migration ou dominations imbriquées. Voir Danièle Kergoat, "Dynamique et consubstantialité des rapports sociaux", op. cit.; Sabah Chaïb, "Femmes immigrées et travail salarié en France", op. cit.; Annette Goldberg Salinas (1996), «Femmes en migration. Une réflexion sur l'état de la question en France", in Migration-Formation, n ${ }^{\circ}$ 105, p. 31-44 ; Faïza Guélamine, "Les faces cachées de la 'différence culturelle'.

La construction d'une altérité ambiguë par les travailleurs sociaux", in Les Cahiers du Cériem, n 8, 2001, p. 9-22.

25. Françoise Gaspard, "Le foulard de la dispute", in Dominique Fougeyrollas-Schwebel, Eleni Varikas (dir.), Féminisme(s). Recompositions et mutations, Cahiers du genre, $\mathrm{n}^{\circ}$ hors- série, 2006, p. 74.

26. Par exemple, si Christine Delphy se penche sur le traitement politique du voile ainsi que sur l'imbrication entre sexisme et racisme, elle n'a, en revanche, pas intégré le travail des migrantes domestiques dans sa théorie du "mode de production domestique". Voir Christine Delphy, L'Ennemi principal. Penser le genre, op. cit.

27. Comme dans le texte d'Éléonore Lépinard, "Malaise dans le concept. Différence, identité et théorie féministe", in Cahiers du genre, vol. 2, n 39, 2005, pp. 107-135.

28. Germaine Tillion, Le Harem et les Cousins, Paris, Le Seuil, 1966.

29. Lila Abu-Lughud, "Feminist longings and postcolonial conditions", op. cit.

30. L'analyse féministe aurait pu poser la question de l'inscription de cette loi dans la continuité des discriminations légales touchant les migrant(e)s et leurs descendants avec ou sans papiers français.

31. Mohamed Houbbaida, "Le costume féminin en milieu rural. Observations préliminaires sur le Maroc précolonial", in Dalenda Largueche (dir.), Histoire des femmes au Maghreb, culture matérielle et vie quotidienne, Tunis, Centre de publication universitaire, 2000.

32. Jacques Berque, Le Maghreb entre deux guerres, Paris, Seuil, 1962.

33. Fatima Mernissi, "Développement capitaliste et perception des femmes dans la société arabo-musulmane : une illustration des paysannes du Gharb, Maroc", art. cit.

34. Fatima Mernissi, Beyond the Veil. Male-Female Dynamics in Muslim Society, London, Al Saqi Books, 1975 ; "Développement capitaliste et perception des femmes dans la société arabo-musulmane : une illustration des paysannes du Gharb, Maroc", art. cit.

35. Abdallah Baroudi, Maroc, impérialisme et émigration, Paris, Le Sycomore, 1978 ; Abdelmalek Sayad, La Double Absence. Des illusions aux souffrances de l'immigré, op. cit.

36. Nouria Ouali, "Les droits dérivés des femmes immigrées : d'une dépendance tacite à une dépendance institutionnalisé", in Womens's Studies, newsletter 5, 1993 ; Sabah Chaïb, "Femmes immigrées et travail salarié en France", op. cit.

37. Nacira Guénif-Souilamas, Des "beurettes" aux descendantes d'immigrants nord-africains, Paris, Grasset/Le Monde édition poche, 2000. 